\title{
Formation of a Magnetic Soliton Lattice in Copper Metaborate
}

\author{
B. Roessli, ${ }^{1}$ J. Schefer, ${ }^{1}$ G. A. Petrakovskii, ${ }^{2}$ B. Ouladdiaf,${ }^{3}$ M. Boehm, ${ }^{1,3}$ \\ U. Staub, ${ }^{4}$ A. Vorotinov, ${ }^{2}$ and L. Bezmaternikh ${ }^{2}$ \\ ${ }^{1}$ Laboratory for Neutron Scattering, ETH Zurich \& Paul Scherrer Institute, CH-5232 Villigen PSI, Switzerland \\ ${ }^{2}$ Institute of Physics SB RAS, 660036 Krasnoyarsk, Russia \\ ${ }^{3}$ Institut Laue-Langevin, Avenue des Martyrs, 38042 Grenoble, Cedex 9, France \\ ${ }^{4}$ Swiss Light Source, Paul Scherrer Institute, CH-5232 Villigen PSI, Switzerland
}

(Received 28 June 2000)

\begin{abstract}
The magnetic ground state of $\mathrm{CuB}_{2} \mathrm{O}_{4}$ is incommensurate at $T=1.8 \mathrm{~K}$ and undergoes a continuous phase transition to a noncollinear commensurate antiferromagnetic state at $T^{\star} \sim 10 \mathrm{~K}$. Close to $T^{\star}$ higher-order magnetic satellites are observed. Coexistence of long- and short-range magnetic order is observed in both magnetic phases. This suggests that the association of the Dzyaloshinskii-Moriya interaction and anisotropy leads to the formation of a magnetic soliton lattice.
\end{abstract}

DOI: 10.1103/PhysRevLett.86.1885

PACS numbers: $75.25 .+\mathrm{z}, 75.10 . \mathrm{Hk}, 75.30 . \mathrm{Gw}$

Incommensurate magnetic structures are characterized by modulations of their spin arrangements over periods which are long compared to the size of the chemical cell and not commensurate with the underlying lattice [1]. The existence of such magnetic structures in insulators is either due to competitions between exchange interactions or relativistic effects such as spin-orbit coupling. While the former effects can be found accidentally, the latter mechanism depends on lattice symmetry. Relativistic interactions were first considered by Dzyaloshinskii [2] and given a microscopic description by Moriya [3]. The DzyaloshinskiiMoriya (DM) interaction is usually written as the cross product of interacting spins $H_{\mathrm{DM}}=\mathbf{D} \cdot\left(\mathbf{S}_{1} \times \mathbf{S}_{2}\right)$ where $\mathbf{D}$ is the DM vector. The direction of the DM vector is determined by the bond symmetry and its scalar by the strength of the spin-orbit coupling [3]. The antisymmetric DM interaction is given by a term linear in the gradient of the magnetization (Lifshitz invariant [4]) in the Landau thermodynamical potential. The Lifshitz invariant in antiferromagnets favors a magnetic ground state where the magnetization forms a spiral structure. The presence of additional crystal anisotropy distorts the simple helicoidal spin arrangement and the angle evolution of the magnetic moments is described by the sine-Gordon equation for a soliton lattice $[5,6]$. It should be recognized that a soliton corresponds to localized or topological dislocations of a periodic structure which arise when nonlinear forces are present [7]. While the formation of incommensurate structures has often been observed in condensed matter physics [8], and recently in low-dimensional spin $S=1 / 2$ systems as in the spin-Peierls compounds TTF-CuBDT [9] and $\mathrm{CuGeO}_{3}$ [10], the formation of solitons in a threedimensionally ordered magnetic lattice is unusual. To our knowledge, a field-induced magnetic soliton lattice has been observed only for the Dzyaloshinskii-Moriya helimagnet $\mathrm{Ba}_{2} \mathrm{CuGe}_{2} \mathrm{O}_{7}$ [11].

The specific heat and susceptibility data show two magnetic phase transitions at $T_{N}=21 \mathrm{~K}$ and $T^{\star} \sim 10 \mathrm{~K}$.
From magnetization measurements it is concluded that copper metaborate is a weak ferromagnet for $T^{\star} \leq$ $T \leq T_{N}$ [12]. In this Letter we report elastic neutron scattering experiments in copper metaborate $\mathrm{CuB}_{2} \mathrm{O}_{4}$. In the temperature range $10 \leq T \leq 21 \mathrm{~K}$ the magnetic structure is commensurate. Upon lowering the temperature below $T^{\star} \sim 10 \mathrm{~K}$, a second magnetic phase transition toward an incommensurate three-dimensional magnetic order occurs. In the incommensurate phase, but in the vicinity of $T^{\star}$, higher-order satellites appear in the neutron diffraction pattern. In that temperature range, this shows that there results a nonuniform rotation of the magnetic moments along the pitch of the helix. As the DM interaction is allowed by symmetry and non-negligible anisotropy in the basal plane has been observed $[13,14]$, the modulation of the order parameter in copper metaborate along the helix direction is described within the Landau theory of phase transitions by the solution of the sine-Gordon equation [15]. This suggests that copper metaborate is the first compound to spontaneously form, i.e., without external disturbance, a magnetic soliton lattice.

The neutron measurements were performed at the neutron spallation source SINQ of the Paul Scherrer Institute and at the high-flux neutron source of the Institut LaueLangevin. The single crystal used in the present experiment was prepared using ${ }^{11} \mathrm{~B}$ at the Institute of Physics in Krasnoyarsk. $\mathrm{CuB}_{2} \mathrm{O}_{4}$ crystallizes in space group $I \overline{4} 2 d$ $\left(D_{2 d}^{12}\right)$ with lattice constants $a=11.528 \AA$, and $c=$ $5.607 \AA$ [16]. The unit cell contains 12 formula units. The $\mathrm{Cu}^{2+}$ ions occupy two inequivalent positions at $\mathrm{Cu}(A)=$ (site $4 b$, point symmetry $\left.S_{4}, 00 \frac{1}{2}\right)$ and $\mathrm{Cu}(B)=($ site $8 d$, point symmetry $C_{2}, x \frac{1}{4} \frac{1}{8}, x=0.0815$ ), respectively. $\mathrm{Cu}(A)$ is at the center of a square unit formed by four oxygen ions, while $\mathrm{Cu}(B)$ is surrounded by six oxygen ions located at the vertices of a distorted octahedron. From high-resolution neutron powder diffraction we conclude that $\mathrm{CuB}_{2} \mathrm{O}_{4}$ does not undergo any structural phase transition down to $T=1.5 \mathrm{~K}$. Systematic scans in reciprocal 
space allowed magnetic reflections to be found at commensurate Bragg positions for $T=12 \mathrm{~K}$. At this temperature, the observation of forbidden reflections such as $(1,1,0)$ or $(0,0,2)$ shows that the magnetic structure is antiferromagnetic. The magnetic structure is accordingly described by the propagation vector $\vec{k}=0$, so that the magnetic and chemical cells coincide. As the lattice symmetry operation $I$ is also a magnetic translation when $\vec{k}=0$, the relevant irreducible representations of the magnetic structure are those of the point group $\overline{4} 2 \mathrm{~m}$. This point group contains eight elements and has five irreducible representations. Four of them are one dimensional $\left(\Gamma_{1}\right.$, $\Gamma_{2}, \Gamma_{3}$, and $\Gamma_{4}$ ) and one, labeled $\Gamma_{5}$, is two dimensional. The reduction of the induction representation gives $\Gamma_{4 b}=$ $\Gamma_{3}+\Gamma_{4}+2 \Gamma_{5}$ and $\Gamma_{8 d}=\Gamma_{1}+2 \Gamma_{2}+\Gamma_{3}+2 \Gamma_{4}+$ $3 \Gamma_{5}$, respectively. The magnetic modes $\Gamma_{3}$ and $\Gamma_{4}$ of the $4 b$ site correspond to a collinear antiferromagnetic and ferromagnetic ordering along the $z$ axis, respectively. The modes associated with the $\Gamma_{5}$ representation describe a noncollinear magnetic structure with the magnetic moments rotated by $90^{\circ}$ from each other. Similar magnetic modes for site $8 d$ can be deduced again with the help of group theory. From a least-square refinement of a diffraction set consisting of 25 pure magnetic peaks $\left(R_{F}=4 \%\right)$, we find that the magnetic structure of $\mathrm{CuB}_{2} \mathrm{O}_{4}$ can be described as a noncollinear arrangement of both the $\mathrm{Cu}(A)$ and $\mathrm{Cu}(B)$ spins along the diagonals of the tetragonal plane. The $\mathrm{Cu}(A)$ magnetic moments possess a small component $\mu_{z}=0.25 \mu_{B}$ parallel to the $c$ axis which corresponds to an angle of $14^{\circ}$ out of the tetragonal $a b$ plane. Symmetry analysis of the chemical structure of $\mathrm{CuB}_{2} \mathrm{O}_{4}$ indicates that the $\mathrm{DM}$ interaction is allowed, e.g., between the $\mathrm{Cu}(A)$ nearest-neighbor spins. The $\mathrm{DM}$ vector is perpendicular to the tetragonal $a b$ plane of the crystal and, accordingly, favors the noncollinear spin arrangement which is actually observed. This indicates that the DM interaction plays a significant role in forming the magnetic ground state in $\mathrm{CuB}_{2} \mathrm{O}_{4}$. The value of the magnetic moment is $\sim 1 \mu_{B}$ for the $\operatorname{Cu}(A)$ spins at $T=12 \mathrm{~K}$. Within the precision of the present measurements, the $\mathrm{Cu}(B)$ spins are confined within the $a b$ plane and at this temperature have a small magnetic moment $\mu \sim 0.25 \mu_{B}$, as shown in Fig. 1. As $\mathrm{Cu}(A)$ and $\mathrm{Cu}(B)$ magnetic moments do not compensate for each other, a spontaneous ferromagnetic moment equal to $0.1 \mu_{B}$ per formula unit exists at $T=12 \mathrm{~K}$. Upon lowering the temperature below $T^{\star} \sim$ $10 \mathrm{~K}$, the magnetic structure of copper metaborate is best described by a spin-density wave with phase modulation. As determined by diffraction measurements, the value of the magnetic moment associated with the $\mathrm{Cu}(B)$ sites increases rapidly below $T^{\star}$ and is equal to $\mu=0.7 \mu_{B}$ at $T=2 \mathrm{~K}$.

As shown in Fig. 2, the propagation vector $\vec{k}$ is temperature dependent below $T=10 \mathrm{~K}$ and two magnetic satellites appear at symmetrical positions with respect to the commensurate reciprocal lattice points. This shows that the magnetic structure of $\mathrm{CuB}_{2} \mathrm{O}_{4}$ becomes incom-

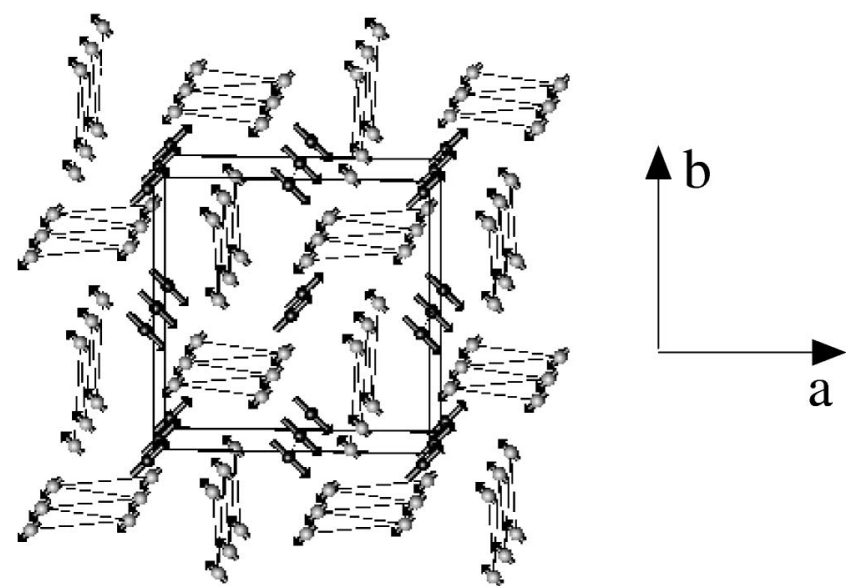

FIG. 1. Antiferromagnetic structure of $\mathrm{CuB}_{2} \mathrm{O}_{4}$ in the commensurate phase. The $\mathrm{Cu}(A)$ and $\mathrm{Cu}(B)$ positions are represented by black and open symbols, respectively, while the lengths and the directions of the arrows mirror the $\mathrm{Cu}^{2+}$ magnetic moments $\vec{\mu}$.

mensurate along the tetragonal axis. The period of the spin modulation continuously increases from $\vec{k}=0$ below $T^{\star} \sim 10 \mathrm{~K}$ to $\vec{k}=(0,0,0.15)$ at $T=1.8 \mathrm{~K}$. At this temperature, the modulation of the spin structure has a period of $c / 0.15 \sim 40 \AA$ along the crystallographic $c$ axis. The evolution of the satellite position with temperature is found to follow a power law

$$
|\vec{k}(T)| \propto\left(T^{\star}-T\right)^{\nu}
$$

with $\nu=0.48$, as shown in Fig. 2 . As $\vec{k}(T)$ smoothly goes to zero with increasing temperature, the period of the helix growths to infinity at $T^{\star} \sim 10 \mathrm{~K}$. In addition, considerable diffuse scattering superimposes on the resolutionlimited Bragg peaks for neutron scattering vectors $\mathbf{Q}$ along the $[0,0,1]$ crystallographic direction, as shown in Fig. 3.

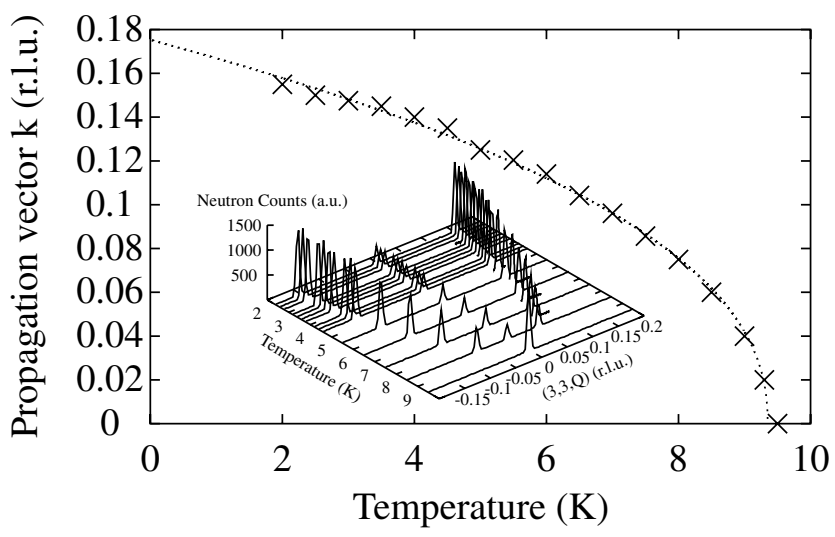

FIG. 2. Temperature dependence of the propagation vector $\vec{k}$. The line is the result of the calculation, as explained in the text. Inset: Neutron elastic scans along $(3,3, Q)$ showing the evolution of the magnetic satellites in $\mathrm{CuB}_{2} \mathrm{O}_{4}$ for selected temperatures. Below $T^{\star}$, the peak at the commensurate position is caused by multiple Bragg scattering. 


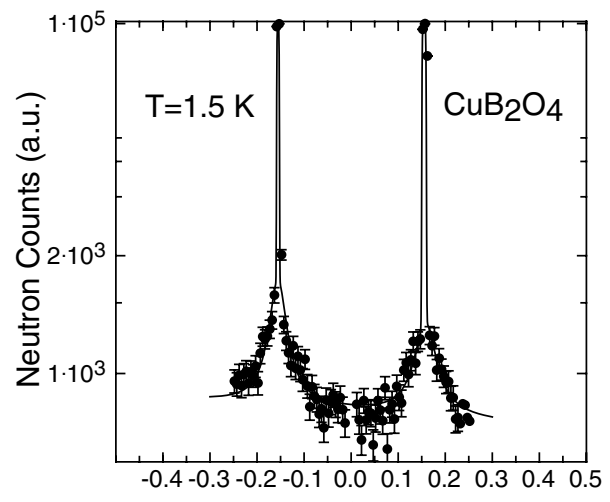

$(1,1, Q)$ (r.l.u.)

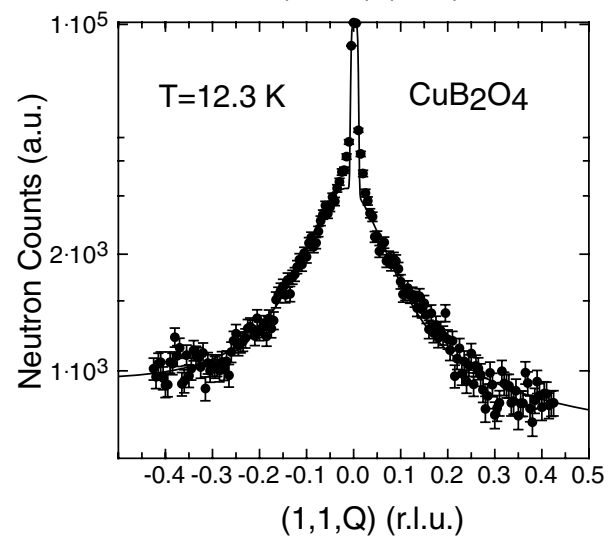

FIG. 3. Elastic and diffuse scattering in $\mathrm{CuB}_{2} \mathrm{O}_{4}$ below and above the incommensurate-commensurate phase transition. Note the logarithmic scale. The line is the result of a Gaussian function and Eq. (2).

The intensity of the diffuse scattering increases with increasing temperature and exhibits a critical divergence close to $T^{\star}$ (Fig. 4), in accordance with the observation of susceptibility and specific heat peaks at this temperature [12]. The line shape of the diffuse scattering is well reproduced by the Fourier transform of the spin correlation function proposed by Ornstein and Zernicke [17]. This function, which is a Lorentzian,

$$
S(\vec{Q})=\frac{A \kappa}{\kappa^{2}+Q^{2}},
$$

is valid only in the temperature range where magnetic fluctuations are large. The average correlation length $\xi$ obtained from the linewidth $\xi=\frac{1}{\kappa}$ yields $\xi \sim 32 \AA$ at $T^{\star}$. $\kappa$ decreases upon passing into the incommensurate magnetic phase and reflects that the correlation length increases with decreasing temperatures up to $\xi \sim 70 \AA$ at $T=$ $1.8 \mathrm{~K}$. We note that diffuse scattering is unexpectedly observed at the lowest temperature reached in this experiment. Namely, for three-dimensionally ordered magnets, critical fluctuations are usually sizable only in a small temperature range below the magnetic phase transition. On the contrary, for spiral structures caused by relativistic interactions, a continuous intensity distribution is expected in a large temperature range, due to perturbations in the simple helix structure [18]. In addition, for a magnetic

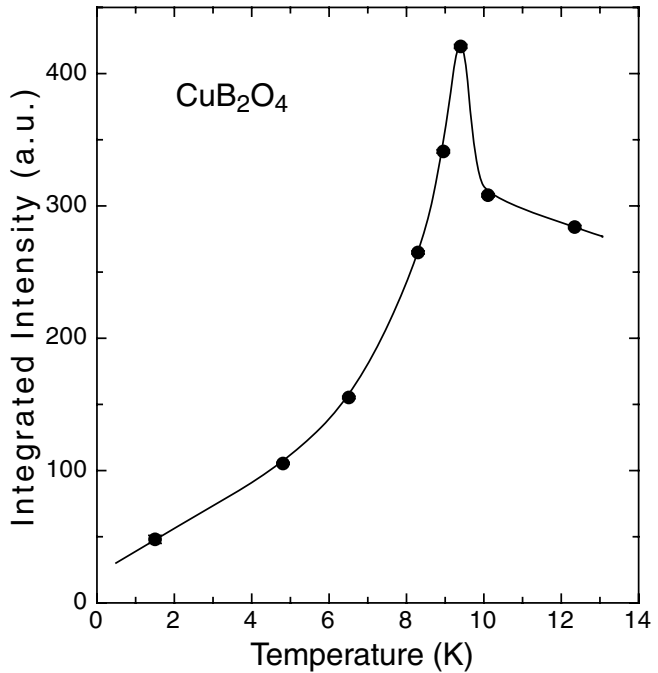

FIG. 4. Temperature dependence of the diffuse scattering in $\mathrm{CuB}_{2} \mathrm{O}_{4}$. Note the increase of intensity in the vicinity of the incommensurate-commensurate phase transition. The line is to guide the eye.

soliton lattice higher-order satellites are expected to appear in the neutron cross section for temperatures near to the incommensurate-commensurate phase transition [19]. Figure 5 shows a neutron scan along the $(1,1, Q)$ direction at $T=9.4 \mathrm{~K}$ which reveals that when the temperature approaches $T^{\star}$, higher-order satellites are produced. We observe that the amplitudes of these higher-order magnetic satellites are largest close to the incommensuratecommensurate phase transition. Therefore, the presence of a multipeak structure in the neutron spectrum with indices $k$ and $3 k$ shows that close to $T^{\star}$, the magnetic structure of copper metaborate can be viewed as a slightly distorted commensurate structure with domain walls.

As the propagation vector $\vec{k}$ decreases to zero when approaching $T^{\star}$, as higher-order magnetic satellites are observed, and also as a commensurate phase transition is induced by applying a magnetic field in the tetragonal plane

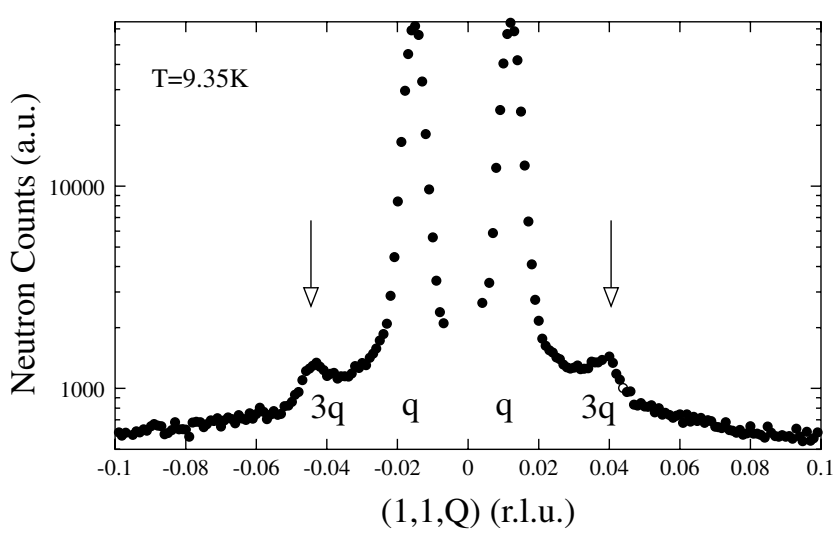

FIG. 5. Neutron elastic scan along the $(1,1, Q)$ direction at $T=9 \mathrm{~K}$ showing the presence of higher-order satellites in addition to the two principal magnetic satellites. The calculated Bragg width due to the instrument resolution and the $10^{\prime}$ mosaic of the sample is $\sim 0.006 \AA^{-1}$. 
$[13,20]$, competing exchange interactions do not appear to be the main driving mechanism of the incommensuratecommensurate phase transition in copper metaborate. The magnetic state of $\mathrm{CuB}_{2} \mathrm{O}_{4}$ is described by a thermodynamical potential which includes two two-component pa- rameters $\left(\nu_{i}, \xi_{i}\right)$ with $i=1,2$, respectively. Considering the symmetry of the crystal $\left(D_{2 d}\right)$ as a whole and noting that the DM interaction is allowed, the Landau thermodynamical potential $(\Phi)$ is given by the sum of exchange, Lifshitz invariant, and anisotropy terms:

$$
\begin{aligned}
\Phi= & \alpha \nu^{2}+\alpha^{A}\left(\nu_{1}^{2}+\xi_{1}^{2}\right)+\alpha^{B}\left(\nu_{2}^{2}+\xi_{2}^{2}\right)+\gamma_{1}\left(\nu_{1} \nu_{2}+\xi_{1} \xi_{2}\right)+\gamma_{2} \nu^{2}\left(\nu_{1}^{2}+\xi_{1}^{2}\right)+\gamma_{3} \nu^{2}\left(\nu_{2}^{2}+\xi_{2}^{2}\right) \\
& +\sigma^{A}\left[\left(\partial \nu_{1} / \partial z\right)^{2}+\left(\partial \xi_{1} / \partial z\right)^{2}\right]+\sigma^{B}\left[\left(\partial \nu_{2} / \partial z\right)^{2}+\left(\partial \xi_{2} / \partial z\right)^{2}\right]+\delta^{A}\left[\left(\partial \nu_{1} / \partial z\right) \xi_{1}-\left(\partial \xi_{1} / \partial z\right) \nu_{1}\right] \\
& +\delta^{B}\left[\left(\partial \nu_{2} / \partial z\right) \xi_{2}-\left(\partial \xi_{2} / \partial z\right) \nu_{2}\right]+\beta \nu^{4}+\beta_{1}^{A}\left(\nu_{1}^{4}+\xi_{1}^{4}\right)+\beta_{2}^{A} \nu_{1}^{2} \xi_{1}^{2}+\beta_{1}^{B}\left(\nu_{2}^{4}+\xi_{2}^{4}\right)+\beta_{2}^{B} \nu_{2}^{2} \xi_{2}^{2}
\end{aligned}
$$

The order parameter $\nu=S_{A 1 z}-S_{A 2 z}$ belongs to the onedimensional representation $\Gamma_{4}$ for the $\mathrm{Cu}(A)$ sublattice. It describes the antiferromagnetic component of the magnetic structure along the $c$ axis. It can be neglected in the analysis of the incommensurate structure and is given here for completeness. $\nu_{1}=\left(S_{A 1 x}+S_{A 1 y}\right)-\left(S_{A 2 x}+S_{A 2 y}\right)$, $\nu_{2}=-\left[S_{B 1 x}+S_{B 2 x}+S_{B 3 x}+S_{B 4 x}\right]+\left[\left(S_{B 1 y}+S_{B 2 y}\right)-\right.$ $\left.\left(S_{B 3 x}+S_{B 4 x}\right)\right]+\left[S_{B 1 z}-S_{B 2 z}+S_{B 3 z}-S_{B 4 z}\right], \quad \xi_{1}=$ $\left(S_{A 1 x}-S_{A 1 y}\right)+\left(S_{A 2 x}-S_{A 2 y}\right), \xi_{2}=\left[\left(S_{B 1 x}+S_{B 2 x}\right)-\right.$ $\left.\left(S_{B 3 x}+S_{B 4 x}\right)\right]+\left[S_{B 1 y}+S_{B 2 y}+S_{B 3 x}+S_{B 4 x}\right]+\left[S_{B 1 z}-\right.$ $\left.S_{B 2 z}-S_{B 3 z}+S_{B 4 z}\right]$ are linear combinations of the magnetic modes. The phenomenological parameters $\alpha, \gamma$, and $\sigma$ are related to the intra- and intersublattice anisotropic exchange interactions. The $\beta_{i}^{j}$ describe the crystalline anisotropy which for a spin $1 / 2$ is of the order of 4 and the $\delta^{j}(i=1,2 ; j=A, B)$ are determined by the strength of the DM interaction. The potential $\Phi$ with the simplifications that $\alpha^{A}=\alpha_{o}^{A}(T-21 \mathrm{~K})$ and $\alpha^{B}=\alpha_{o}^{B}(T-10 \mathrm{~K})$ explains the mechanisms of formation of the magnetic soliton lattice in $\mathrm{CuB}_{2} \mathrm{O}_{4}$. It also turns out from the analysis of the temperature dependence of the magnetization, the susceptibility, and the specific heat data, that the order parameter of the $\mathrm{Cu}(A)$ sublattice depends upon temperature like the sublattice magnetization of an antiferromagnet with critical temperature $T=21 \mathrm{~K}$. On the other hand, calculations show that the order parameter of the $\mathrm{Cu}(B)$ sublattice remains very small in the temperature range $T^{\star}=10 \mathrm{~K}$ to $T=21 \mathrm{~K}$ and rapidly increases for temperatures lower than $T=10 \mathrm{~K}$, in agreement with our diffraction data. The association of the magnetic anisotropy with Lifshitz invariants (we obtain $\delta^{B} \gg \delta^{A}$ ) appears to be at the origin of the magnetic soliton lattice and of the incommensurate phase. A detailed analysis will be published elsewhere [21].

In conclusion, we have shown that copper metaborate forms a magnetic soliton lattice, the origin of which is ascribed to the association of Lifshitz invariants in the thermodynamical potential with magnetic anisotropy of order $n=4$.

We acknowledge useful discussions with Professor V. Zinenko and Professor M. Popov.
[1] For example, T. Chattopadhyay, Science 264, 226 (1994).

[2] I.E. Dzyaloshinskii, Sov. Phys. JETP 19, 960 (1964).

[3] T. Moriya, Phys. Rev. 120, 91 (1960).

[4] E. M. Lifshitz, JETP 11, 253 (1941).

[5] I.E. Dzyaloshinskii, Sov. Phys. JETP 20, 665 (1965).

[6] Yu. A. Izyumov, Physica (Amsterdam) 174B, 9 (1991).

[7] For a review, see, e.g., Solitons and Condensed Matter Physics, edited by A. R. Bishop and T. Schneider (SpringerVerlag, New York, 1978).

[8] For example, P. Bak, Rep. Prog. Phys. 45, 687 (1982).

[9] V. Kiryukhin, B. Keimer, and D.E. Moncton, Phys. Rev. Lett. 74, 1669 (1995).

[10] V. Kiryukhin et al., Phys. Rev. Lett. 76, 4608 (1996); M. Horvatić et al., Phys. Rev. Lett. 83, 420 (1999).

[11] A. Zheludev et al., Phys. Rev. B 54, 15163 (1996); Phys. Rev. Lett. 78, 4857 (1997); Phys. Rev. B 57, 2968 (1998); 81, 5410 (1998); Phys. Rev. B 59, 11432 (1999).

[12] G. Petrakovskii et al., J. Magn. Magn. Mater. 205, 105 (1999).

[13] G. A. Petrakovskii et al., Phys. Solid State 42, 321 (2000).

[14] A. Pankrats et al., Phys. Solid State 42, 96 (2000).

[15] J.C. Tolédano and P. Tolédano, The Landau Theory of Phase Transitions, World Scientific Lecture Notes in Physics Vol. 3 (World Scientific, Singapore, 1987).

[16] M. Martinez-Ripoli et al., Acta Crystallogr. Sect. B 27, 677 (1971).

[17] M.F. Collins, Magnetic Critical Scattering (Oxford University Press, New York, 1989).

[18] Yu. A. Izyumov and V. M. Laptev, Sov. Phys. JETP 62, 755 (1986); Yu. A. Izyumov, Neutron Diffraction in LongPeriodic Structures (Energoatomizdat, Moscow, 1987).

[19] Yu. A. Izyumov, Sov. Phys. Usp. 27, 845 (1985).

[20] First analysis of the spin waves shows that the exchange parameters are of comparable strength along the main crystallographic directions. Application of a magnetic field in the tetragonal plane induces a commensurate phase at $H_{c}=1.3 \mathrm{~T}$ and $T=4.2 \mathrm{~K}$. This behavior is predicted for a magnetic soliton lattice [18].

[21] G. A. Petrakovskii et al. (to be published). 